\title{
Gut Bacterial Diversity of Insecticide-Susceptible and -Resistant Nymphs of the Brown Planthopper Nilaparvata lugens Stål (Hemiptera: Delphacidae) and Elucidation of Their Putative Functional Roles ${ }^{\text {s }}$
}

\author{
Vijayakumar M. Malathi ${ }^{1}$, Ravi P. More ${ }^{1}$, Rangasamy Anandham ${ }^{2}$, Gandhi R. Gracy ${ }^{1}$, Muthugounder Mohan ${ }^{1}$, \\ Thiruvengadam Venkatesan ${ }^{1}$, Sandipan Samaddar ${ }^{3}$, Sushil K. Jalali ${ }^{1 *}$, and Tongmin $\mathrm{Sa}^{3 *}$ \\ ${ }^{1}$ Biotechnology Laboratory, Department of Molecular Entomology, ICAR-National Bureau of Agricultural Insect Resources, Bengaluru 560024 , \\ Karnataka, India \\ ${ }^{2}$ Department of Agricultural Microbiology, Agricultural College and Research Institute, Tamil Nadu Agricultural University, Madurai 625 \\ 104, India \\ ${ }^{3}$ Department of Environmental and Biological Chemistry, Chungbuk National University, Cheongju 28644, Republic of Korea
}

Received: November 22, 2017

Revised: April 8, 2018

Accepted: April 14, 2018

First published online April 14, 2018

*Corresponding authors

Tongmin Sa

Phone: +82-43-261-2561;

Fax: +82-43-271-5921;

E-mail: tomsa@chungbuk.ac.kr

Sushil Kumar Jalali

Phone: +91 (80) 23511981;

Fax: +91 (80) 23411961;

E-mail: jalalisk@gmail.com

S upplementary data for this paper are available on-line only at http://jmb.or.kr.

pISSN 1017-7825, eISSN 1738-8872

Copyright(C) 2018 by

The Korean Society for Microbiology and Biotechnology
Knowledge about the gut bacterial communities associated with insects is essential to understand their roles in the physiology of the host. In the present study, the gut bacterial communities of a laboratory-reared insecticide-susceptible (IS), and a field-collected insecticide-resistant (IR) population of a major rice pest, the brown planthopper Nilaparvata lugens, were evaluated. The deep-sequencing analysis of the V3 hypervariable region of the 16S rRNA gene was performed using Illumina and the sequence data were processed using QIIME. The toxicological bioassays showed that compared with the IS population, IR population exhibited 7.9-, 6.7-, 14.8-, and 18.7-fold resistance to acephate, imidacloprid, thiamethoxam, and buprofezin, respectively. The analysis of the alpha diversity indicated a higher bacterial diversity and richness associated with the IR population. The dominant phylum in the IS population was Proteobacteria (99.86\%), whereas the IR population consisted of Firmicutes $(46.06 \%)$, followed by Bacteroidetes $(30.8 \%)$ and Proteobacteria (15.49\%). Morganella, Weissella, and Enterococcus were among the genera shared between the two populations and might form the core bacteria associated with $N$. lugens. The taxonomic-tophenotypic mapping revealed the presence of ammonia oxidizers, nitrogen fixers, sulfur oxidizers and reducers, xylan degraders, and aromatic hydrocarbon degraders in the metagenome of $N$. lugens. Interestingly, the IR population was found to be enriched with bacteria involved in detoxification functions. The results obtained in this study provide a basis for future studies elucidating the roles of the gut bacteria in the insecticide resistanceassociated symbiotic relationship and on the design of novel strategies for the management of N. lugens.

Keywords: Gut bacterial community, Illumina MiSeq, insecticide resistance, Nilaparvata lugens, $16 \mathrm{~S}$ rRNA, deep sequencing

\section{Introduction}

The brown planthopper (BPH) Nilaparvata lugens (Stål) (Hemiptera: Delphacidae) is one of the most economically important pests of rice throughout the tropical and temperate regions of Asia. This monophagous pest sucks the phloem sap of rice plants, causing the characteristic stunting, wilting, and browning of the crop, which is often referred to as "hopper burn" [1]. The BPH also serves as a vector of the rice grassy stunt virus and the rice ragged 
stunt virus [2]. In recent years, BPH infestations have intensified across Asia, thereby posing severe threats to rice production, and the losses due to the $\mathrm{BPH}$ infestations have been estimated to be more than 300 million USD per annum [2]. Control of the $\mathrm{BPH}$ relies primarily on the application of chemical insecticides. However, most chemistries used against this pest so far have become compromised by insecticide resistance [3]. The evolution of resistance to insecticides by various insect pests is a serious problem across the world and their mechanisms have been attributed to the increased activity of the insecticide detoxifying enzymes and/or insensitive target sites [4]. Recent studies have reported the role of symbiotic bacteria of the insect gut in imparting resistance to insecticides [5, 6]. The development of efficient alternative control strategies is essential for the effective management of the $\mathrm{BPH}$.

The insect gut harbors diverse communities of microorganisms with interactions ranging from obligate mutualism to facultative parasitism [7]. The host gut offers a highly nutrient-rich niche for the survival of the microbes, and in return, the microbes provide many beneficial services to their insect hosts [8]. For instance, the bacterium Buchnera is known to provide its aphid hosts with essential amino acids [9]. Furthermore, the associated microbiota in the hindgut of termites is known to play a role in the degradation of dietary celluloses [10]. The symbiotic bacteria have been suggested to assist their insect hosts by degrading the ingested compounds, which are otherwise toxic and may mediate the evolution of resistance to insecticides [11]. Recently, a gut bacterium, Citrobacter freundii of the oriental fruit fly, has been demonstrated to degrade the organophosphate trichlorfon into chloral hydrate and dimethyl phosphate, thereby enhancing the host resistance to trichlorfon [12]. Given that the resistance to insecticides is a major obstacle to the control of insect pests worldwide, it is essential to understand the possibility of the bacterial symbiont-mediated insecticide resistance. Such studies may enable novel strategies for pest control as well as the prospective biotechnological applications, including bioremediation [13]. A comprehensive cataloging of the bacterial flora in the guts of insecticide-susceptible and insecticide-resistant insect populations is a prerequisite for the better understanding of the candidate bacteria involved and for elucidating the mechanism of the bacterial symbiont-mediated resistance to insecticides.

Both culture-dependent and culture-independent techniques have been widely used in the characterization of the bacterial communities associated with the insect gut. The selectivity of the nutrient media and the culture conditions results in the enumeration of only a fraction of the microbial community and, hence, the culture-dependent techniques may not reflect the actual number of microbes and their composition in a given sample. Since the early 2000s, culture-independent molecular fingerprinting techniques, such as terminal-restriction fragment length polymorphism, denaturing gradient gel electrophoresis, and temporal temperature gradient gel electrophoresis, have been widely used to profile the bacterial diversity associated with the insect gut $[11,14,15]$. Although these techniques are more robust than culturing, they do not provide an accurate picture of the complexity and relative abundance of the bacterial communities associated with the insect gut, owing to their low throughput and semi-quantitative nature. Although 16S rRNA gene cloning and sequencing is a less biased approach that is widely used to identify insect gut bacteria, it is highly time-consuming [16]. On the other hand, the recent advancements in DNA sequencing technologies offer a comprehensive identification of the bacterial communities associated with various ecological niches, including the insect gut $[6,17]$. Unprecedented data obtained using the next-generation sequencing technologies provide thorough knowledge of the complex microbial communities with high coverage and depth.

In the present study, ultra-deep sequencing of the V3 hypervariable region of the $16 \mathrm{~S}$ rRNA gene was used to investigate the composition and diversity of the gut bacteria associated with N. lugens. A comparative catalog of the gut bacteria associated with the insecticide-susceptible (IS) and insecticide-resistant (IR) populations of N. lugens is presented, and the variations in the bacterial community structure are discussed in terms of the possible roles of the bacterial flora in imparting insecticide resistance to the host. Furthermore, putative functional profiling was performed on the basis of the taxonomic diversity, which enabled us to get preliminary knowledge of the symbiotic relationship between $N$. lugens and its gut bacteria.

\section{Materials and Methods}

\section{Insect Populations}

The IS population of the BPH was obtained from the Rice Entomology Section, Zonal Agricultural Research Station (ZARS), Mandya, Karnataka, India $\left(12^{\circ} 31^{\prime} \mathrm{N}, 76^{\circ} 53^{\prime} \mathrm{E}\right)$, where the population was maintained under laboratory conditions (in 20-25-day-old seedlings of Taichung Native 1 (TN1), a rice variety susceptible to the $\mathrm{BPH}$, cultured in wooden cages $(70 \times 62 \times 75 \mathrm{~cm})$, at $28 \pm 1^{\circ} \mathrm{C}$ and a 16:8 h L:D) for more than 10 years, without exposure to any insecticides.

The IR population used in the present study was originally 
collected during December 2013 from a rice field in Tiruchirappalli, Tamil Nadu, India $\left(10^{\circ} 86^{\prime} \mathrm{N}, 78^{\circ} 53^{\prime} \mathrm{E}\right)$, where insecticides are heavily sprayed to contain the BPH infestation. The field-collected adults or nymphs were maintained under laboratory conditions as mentioned above and mass mated to obtain the F1 generation to assess the levels of their resistance to various insecticides, compared with the IS population.

\section{Insecticides and Toxicological Bioassay}

In order to determine the resistance status of the field-collected $\mathrm{BPH}$, the five most commonly used commercial-grade insecticides acephate (Asataf 75\% SP), imidacloprid (Confidor 17.8\% SL), thiamethoxam (Actara 25\% WG), buprofezin (Applaud 25\% SC), and etofenprox (Bombard 10\% EC) were used.

To assay the dose response of the BPH to the insecticides, the rice stem-dipping method was used [18]. Briefly, a total of 10-15 early fifth instar nymphs of the BPH were introduced into $500 \mathrm{ml}$ plastic cups, each containing basal 10-cm-long, 50-60-day-old rice stems with their root portions placed on a mixture of vermicompost, sand, and water. The cups were secured with a muslin cloth, tied at the mouth of each cup with a rubber band. The rice stems were dipped in appropriate dilutions of each of the five insecticides. A total of three replicates per dose of each of the 5-7 dilutions of the insecticides were used. Rice stems dipped in distilled water were used as the control. Mortality was recorded after $72 \mathrm{~h}$ of the treatment. The nymphs were considered dead if they failed to move after gentle prodding with a fine brush.

Mortality data were subjected to probit analysis for determining the lethal concentration $\left(\mathrm{LC}_{50}\right)$ values and their $95 \%$ confidence limits (CLs) using the POLO plus program (LeOra software 2002, USA). The classification of the levels of resistance was performed on the basis of the resistance factor (RF) value at the $\mathrm{LC}_{50}$ level: $\mathrm{RF}$ value $<10$-fold as low resistance, $R F$ value $=10-40$-fold as moderate resistance, $R F$ value $=40-160$-fold as high resistance, and $R F$ value $>160$-fold as extremely high resistance, where $\mathrm{RF}=\mathrm{LC}_{50}$ of the field-collected IR population/ $\mathrm{LC}_{50}$ of the laboratory-reared IS population [19].

\section{Extraction of DNA from the Guts of the IS and IR BPH}

A total of 30 early fifth instar nymphs (male and female) were selected from each of the two populations and surface-sterilized in $75 \%$ ethanol. For the IR population, the nymphs selected from the F1 generation were dissected and their gut regions were transferred to sterile microcentrifuge tubes. The DNA was extracted separately from the guts of the nymphs of each population, using the Qiagen DNeasy Blood and Tissue Kit (Qiagen, Germany), following the manufacturer's protocol. The extracted DNA was quality checked by electrophoresis on a $0.8 \%$ agarose gel, stained with ethidium bromide, and quantified using a Qubit fluorometer (Invitrogen, USA). The extracted DNA was stored at $-80^{\circ} \mathrm{C}$ until used. The DNA extracted from the nymphs of each population was pooled and a total of two samples were selected for amplicon library construction.

\section{Amplicon Library Construction and Illumina Sequencing}

The V3 amplicons for the deep sequencing in the Illumina MiSeq platform were generated using a two-step amplification procedure. The initial reaction targeted the V3 hypervariable region of the $16 \mathrm{~S}$ rRNA gene, using the following primers: 341F $5^{\prime}$ CCTACGGGAGGCAGCAG-3' and 518R 5'-ATTACCGCGGCT GCTGG-3'. Reactions were performed in a C1000 thermal cycler (Bio-Rad Laboratories Inc., USA) with the 2X KAPA HiFi HotStart ready mix (KAPA Biosystems, USA) and $120 \mathrm{ng}$ of the gut DNA as template. An initial denaturation at $95^{\circ} \mathrm{C}$ for 2 min was followed by 20 cycles, each consisting of denaturation at $95^{\circ} \mathrm{C}$ for $1 \mathrm{~min}$, annealing at $55^{\circ} \mathrm{C}$ for $1 \mathrm{~min}$, and a final extension at $72^{\circ} \mathrm{C}$ for $5 \mathrm{~min}$. The amplified products ( 200 bp) were visualized by electrophoresis on a $1.5 \%$ agarose gel, stained with ethidium bromide, and purified by gel elution, using the MiniElute PCR Purification Kit (Qiagen, Germany). Subsequently, an index PCR was performed in which the Illumina MiSeq adapters and the indices with unique barcodes for multiplexing were added to 200 ng of the purified PCR products, as suggested previously [20]. The PCR conditions were the same as described above except that the number of cycles was reduced to 10 . The amplicons were cleaned up using Agencourt AMPure XP SPRI beads (Beckman Coulter Inc., USA). The libraries were quality checked by running an aliquot on an Agilent High Sensitivity Bioanalyzer chip (Agilent Technologies, USA) and quantified using the Qubit fluorometer.

Using the paired-end strategy on an Illumina MiSeq Sequencer at M/s Genotypic Technologies (India), the barcoded PCR products were combined in equal quantities (ng) into a single multiplexed sample and sequenced. The Illumina pipeline (ver. 2.2) was used for the image analysis and base calling.

\section{Processing of the Sequence Reads and Data Analysis}

The Consensus Assessment of Sequence and Variation (CASAVA) was used for demultiplexing. The raw reads were quality checked using FastQC. The adapter sequences and the low-quality bases were removed using the adapter base length (abl)-trimming Perl script (Genotypic proprietary tool). Using fastq-join, the processed paired-end reads with $75 \%$ of the high-quality bases $(Q \geq 30)$ were stitched with a minimum overlap of $8 \mathrm{bp}$. The stitched reads were analyzed using QIIME software [21]. The closed-reference operational taxonomic units (OTUs) picking approach was applied using the UCLUST-ref method in the QIIME pipeline, wherein the clustered query sequences were mapped with $\geq 97 \%$ sequence similarity against the curated, chimera-free, 16S rRNA database Greengenes (ver. 13.8). The OTU table generated in .biom format was then normalized to an even sampling depth of 0.7 million reads using random sampling (without replacement) with single_rarefaction.py in the QIIME pipeline. The singletons were removed. The alpha diversity was assessed, applying various metrices (i.e., Shannon, Simpson, Chao1, and the observed OTUs), and a rarefaction plot was generated. 
Table 1. Dose-responses of two populations of Nilaparvata lugens to various insecticides.

\begin{tabular}{lcccc}
\hline Insecticide & Population & Slope \pm SE & LC $_{50}(95 \% \text { CL })^{\mathrm{a}}$ & LCR $_{50}(95 \% \mathrm{CL})^{\mathrm{b}}$ \\
\hline Acephate & IS & $0.872 \pm 0.104$ & $0.077(0.049-0.117)$ & $7.87(4.347-14.285)^{*}$ \\
Imidacloprid & IR & $1.209 \pm 0.160$ & $0.608(0.398-0.920)$ & $6.71(3.472-12.987)^{*}$ \\
& IS & $0.779 \pm 0.129$ & $0.044(0.023-0.070)$ & \\
Thiamethoxam & IR & $1.274 \pm 0.182$ & $0.297(0.209-0.463)$ & $14.92(6.134-37.037)^{*}$ \\
Buprofezin & IS & $0.887 \pm 0.176$ & $0.014(0.004-0.026)$ & $18.51(8.474-41.666)^{*}$ \\
Etofenprox & IR & $1.669 \pm 0.257$ & $0.208(0.147-0.312)$ & \\
& IS & $0.603 \pm 0.075$ & $0.122(0.067-0.208)$ & $1.57(0.984-2.512)$ \\
\hline
\end{tabular}

${ }^{\mathrm{a}} \mathrm{The} \mathrm{LC}_{50}$ values are expressed as $\mathrm{mg}$ of ai/ $\mathrm{L}$ for acephate and thiamethoxam and $\mathrm{ml}$ of ai/ $\mathrm{L}$ for imidacloprid, buprofezin, and etofenprox.

${ }^{\mathrm{b}}$ Lethal concentration ratios at $\mathrm{LC}_{50}$ and their $95 \% \mathrm{CL}$ were calculated by the POLO plus program.

${ }^{*} \mathrm{LCR}_{50}$ is significant $(\alpha=0.05)$ if the $95 \%$ CL does not include 1.0 .

The Statistical Analysis of Metagenomic Profiles (STAMP) software package (ver. 2.0.9) was used to explore the significantly abundant orders between the IS and IR populations [22]. The significance testing was performed by applying the G-test with Yates' continuity correction and Fisher's exact test with absolute effective size statistics; that is, the difference between proportions (DP) without any correction method. Using the standard asymptotic approach, the $95 \%$ confidence intervals (CI) for the DP statistic were constructed. The unclassified reads were removed from the dataset and the bacterial orders having a value of $p>0.05$ were filtered and the remaining features were considered significant for the study.

In order to assess the putative functional profiles, a web-based analytical pipeline for comparative metagenomic studies, the METAGENassist, which performs the automated taxonomic-tophenotypic mapping, was used [23]. The taxonomic abundance data were used as the input files. Row-wise normalization by sum was performed between the samples, and the data were analyzed for the "metabolism by phenotype." The results obtained were further processed with the ClustVis web tool, in order to generate the heatmaps [24]. Using the mvabund package in the R software, significant differences in the putative functional profiles between the IS and IR populations were determined $[25,26]$.

\section{Submission of the Sequencing Data}

The paired-end Illumina sequence reads from this study were deposited at the Sequence Read Archive database under the accession number SRP075392.

\section{Results}

\section{Toxicological Bioassay}

The $\mathrm{LC}_{50}$ values of the IS and IR populations for the tested insecticides are presented in Table 1 . The $\mathrm{LC}_{50}$ values for acephate, imidacloprid, thiamethoxam, and buprofezin of the IR population varied significantly from those of the IS population. However, no significant difference in the $\mathrm{LC}_{50}$ values of both populations was observed for etofenprox. Based on the RF values, the IR population was found to show low levels of resistance to acephate (RF value $=7.9)$ and imidacloprid (RF value $=6.7)$, and moderate levels of resistance to thiamethoxam $(\mathrm{RF}$ value $=14.8)$ and buprofezin $($ RF value $=18.7)$

\section{Illumina MiSeq Metagenomic Data and Taxonomic Assignments}

A total of 918,522 and 2,129,864 paired-end reads were obtained from the metagenomes of the IS and IR populations, respectively. After quality filtering (Table 2), the reads were

Table 2. Read count statistics for the insecticide-susceptible (IS) and insecticide-resistant (IR) metagenomes.

\begin{tabular}{|c|c|c|c|c|c|c|}
\hline Sample & $\begin{array}{c}\text { Total paired } \\
\text { end reads }\end{array}$ & $\begin{array}{l}\text { Processed } \\
\text { reads }\end{array}$ & $\begin{array}{l}\text { Total identified } \\
\text { rRNA sequences }\end{array}$ & $\begin{array}{c}\text { Total OTUs picked after } \\
\text { rarefaction at } 0.7 \text { million reads }\end{array}$ & $\begin{array}{l}\text { Total reads after } \\
\text { singleton removal }\end{array}$ & $\begin{array}{c}\text { Good's coverage index }{ }^{\mathrm{a}} \\
(\%)\end{array}$ \\
\hline IS & 918,522 & 781,520 & 758,788 & 525 & 699,720 & 99.96 \\
\hline IR & $2,129,864$ & $1,878,549$ & $1,487,243$ & 3,190 & 698,691 & 99.87 \\
\hline
\end{tabular}

${ }^{\mathrm{a}}$ Goods coverage calculated on the rarefied biom file at a depth of 0.7 million reads. 


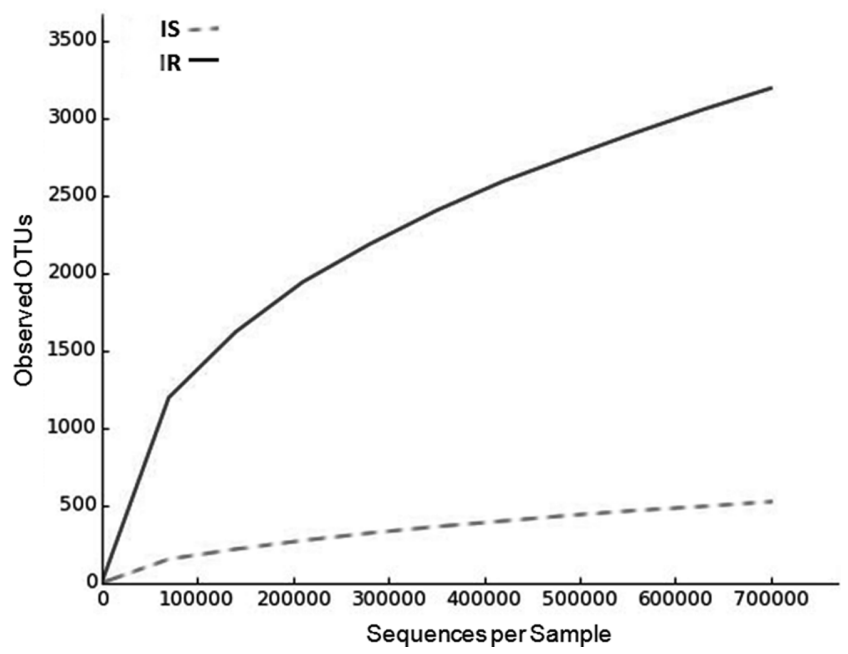

Fig. 1. Rarefaction curves of the gut metagenome sequences of insecticide-susceptible (IS) and -resistant (IR) populations of Nilaparvata lugens at a depth of 0.7 million reads based on the comparison of datasets in the Green Genes database.

rarefied at a depth of 0.7 million reads and a total of 525 and 3,190 OTUs were picked for the IS and IR populations, respectively. Furthermore, the removal of the singletons yielded 245 and 1,881 OTUs for the IS and IR populations, respectively. The rarefaction curve (OTUs at $97 \%$ identity) is presented in Fig. 1. The Good's coverage index of the rarefied biom at a depth of 0.7 million reads is presented in Table 2. The Chao1 index, which reflects the richness of species within a single sample along with the Shannon and Simpson diversity indices, indicated a higher bacterial diversity associated with the IR population (Table 3).

The taxonomic assignment of the metagenomic data was performed at the various levels of classification. A total of 9 and 23 phyla were detected in the IS and IR populations, respectively. Variations were observed in the relative abundance of the bacterial phyla between the IS and IR populations. In the IR population, the highest number of sequences were annotated to the phylum Firmicutes (46.06\%), followed by Bacteroidetes (30.80\%) and Proteobacteria

Table 3. Richness and diversity indices estimation for the rarefied biom file of insecticide-susceptible (IS) and insecticideresistant (IR) populations based on alpha diversity analysis.

\begin{tabular}{ccccc}
\hline Samples & Shannon & Simpson & Chao1 & $\begin{array}{c}\text { Observed } \\
\text { species }\end{array}$ \\
\hline IS & 1.55 & 0.552 & $1,125.92$ & 525 \\
IR & 5.47 & 0.924 & $5,340.96$ & 3,190 \\
\hline
\end{tabular}

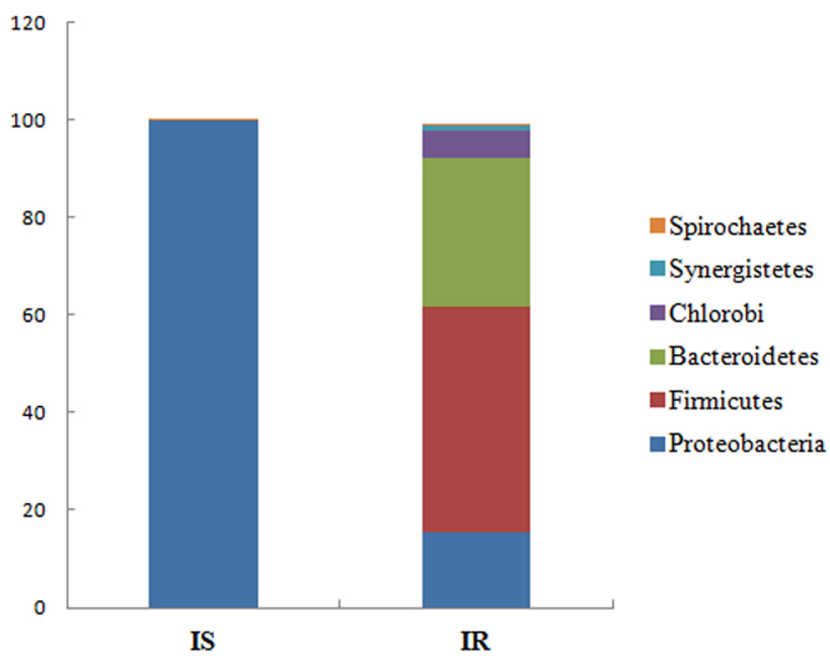

Fig. 2. Relative abundance of bacterial phyla in insecticidesusceptible (IS) and -resistant (IR) populations of Nilaparvata lugens.

The percentage of sequences is plotted on the Y-axis.

$(15.49 \%)$. On the other hand, in the IS population, almost all the sequences were annotated to Proteobacteria (99.86\%), and a few sequences belonged to Firmicutes $(0.10 \%)$ and Bacteroidetes (0.03\%) (Supplementary File S1). The stacked bar plot showing the relative abundance of the top six phyla from the rarefied biom in the IS and IR populations is presented in Fig. 2.

The differentially abundant bacterial orders for the metagenomes of the IS and IR populations were identified by STAMP analysis (Fig. 3). Bacteroidales, Enterobacteriales, Lactobacillales, Clostridiales, Burkholderiales, and Bacillales were among the orders significantly abundant in the IR population, whereas Rhodospirillales was abundant in the IS population.

At the genus level, $98.94 \%$ of the reads in the IS population were assigned to an unclassified genus, $0.30 \%$ of the reads were assigned to Rhodovarius, $0.12 \%$ of the reads were assigned to Paracraurococcus, and $0.11 \%$ of the reads were assigned to Roseomonas, all belonging to the family Acetobacteraceae. In the IR population, $24.85 \%$ of the reads belonged to an unclassified genus of the order Bacteroidales, $23 \%$ of the reads were assigned to the genus Trichococcus, $5.57 \%$ of the reads were assigned to Prosthecochloris, and $2.78 \%$ of the reads were assigned to Clostridiisalibacter. The assigned genera (abundance count $\geq 100$ reads) were considered for identifying the dominant, common, and unique genera from the IS and IR populations (Supplementary File S2). The reads assigned to the unclassified genera were 

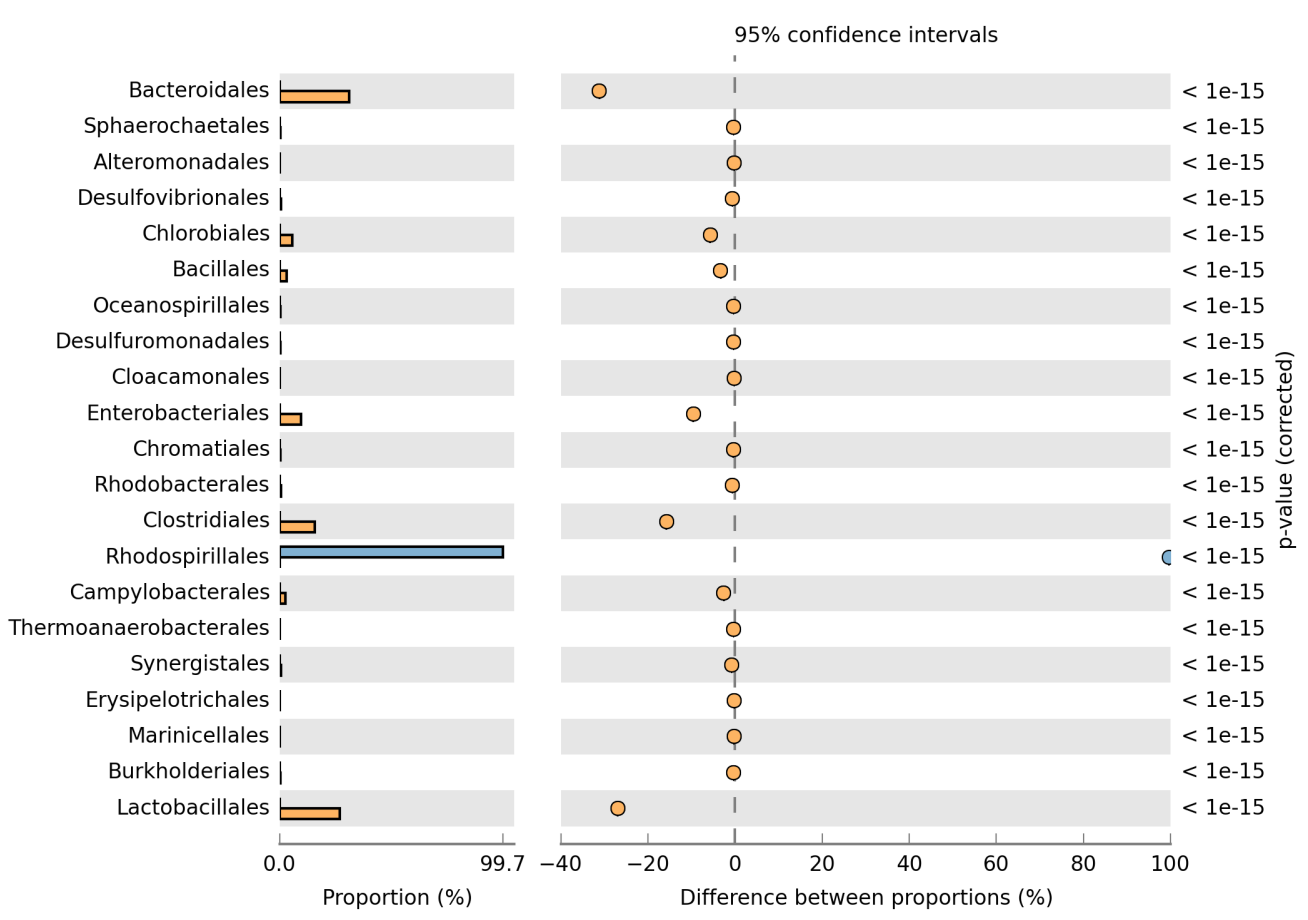

Fig. 3. Extended bar plot in STAMP, illustrating the significant abundance of bacterial orders in the metagenomes of insecticidesusceptible (IS) and -resistant (IR) populations of Nilaparvata lugens.

Orders with abundance count $\geq 1,000$ were filtered and analyzed. Corrected $p$-values were calculated based on the G-test (with Yates' continuity correction) and Fisher's exact test. Orders with $p$-value $<0.05$ were considered significant and were retained. A positive difference between the proportions indicates a significant abundance of genera in the IS-data set, whereas a negative difference between proportions shows a significant abundance of genera in the IR-data set.

not considered. Among the 10 genera from the IS population and the 72 genera from the IR population, only three genera (Morganella, Weissella, and Enterococcus) were found to be shared between the two populations.

Paracraurococcus, Roseomonas, Swaminathania, and Acetobacter were among the unique genera associated with the IS population. Alkalibacterium, Solibacillus, Dorea, Marinicella, Natronobacillus, Allochromatium, and Arcobacter were the genera found exclusively in the IR population. The list of the dominant and the rare, unique genera associated with the IS and IR populations are presented in Supplementary File S3.

\section{Taxonomy-Based Functional Profiling}

The taxonomic-to-phenotypic mapping revealed the operation of processes such as ammonia oxidation, nitrogen fixation, sulfur oxidation and reduction, dehalogenation, and the degradation of xylans as well as aromatic hydrocarbons in the gut of $N$. lugens. However, differences in the relative abundance of the microbiota involved in these processes were recorded between the two populations (Fig. 4). In the IR population, the nitrogen fixers, as well as the sulfur oxidizers and reducers, were relatively more abundant compared with the IS population. Similarly, the microbiota performing processes such as dehalogenation, the degradation of chitin as well as aromatic hydrocarbons, and the metabolism of atrazine were abundant in the IR population compared with their IS counterparts. Conversely, the bacterial flora performing processes such as the oxidation of ammonia and methane as well as the reduction of sulfate were relatively more abundant in the IS population. The cellulose degraders and the alkane degraders were found exclusively in the IR samples, whereas the acidproducing bacteria were present in the IS population only. The statistical analysis showed significant differences in the putative functional profiles $(p=0.001)$ between the IS and IR populations.

\section{Discussion}

Knowledge of the microbial community structure within an insect host is a prerequisite for understanding the complexities as well as the relationship dynamics and the types of interactions between the two. Previously, Tang et 


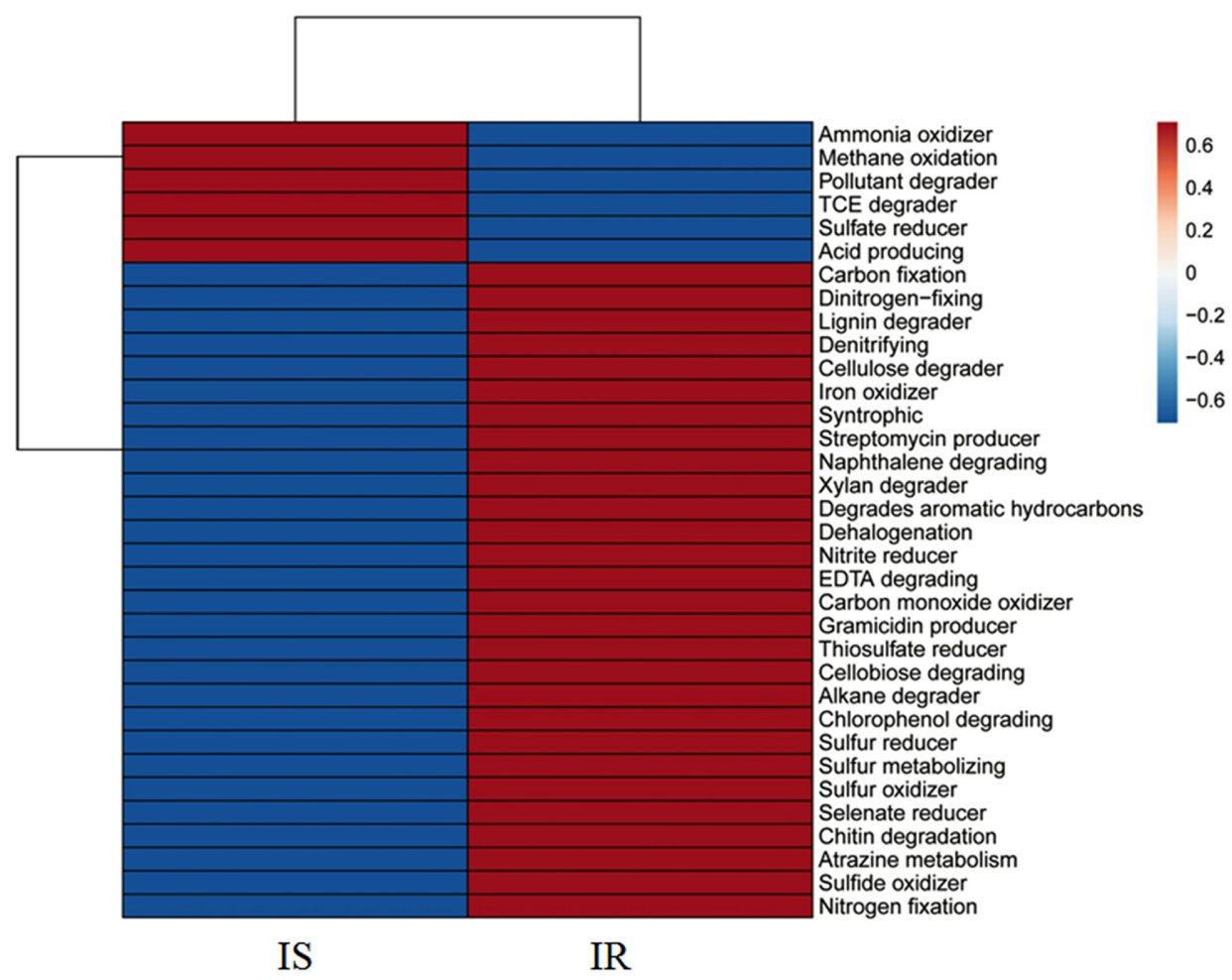

Fig. 4. Heatmap representing putative functional profiles of gut metagenomes of the two populations of Nilaparvata lugens. Annotations were performed using the METAGENassist server, and the heatmap was generated using ClustVis software

al. [16] studied the bacterial flora associated with the different biotypes of $N$. lugens by $16 \mathrm{~S}$ rRNA gene cloning analysis, and identified a total of 18 OTUs. Employing the denaturing gradient gel electrophoresis technique, $\mathrm{Xu}$ et al. [27] reported the bacterial communities from the different geographic and resistant virulent populations of N. lugens. To the best of our knowledge, this is the first study providing a comparative analysis of the bacterial communities associated with the IS and IR populations of N. lugens, an important pest of rice. The identification and characterization of the bacterial flora exclusively associated with the IR population of $N$. lugens may enable novel approaches to manage the resistance to the insecticides and economic crop production.

In the present study, distinctive differences in the richness and diversity of the gut microbiota were observed among the IS and IR populations. The alpha diversity analysis showed that the gut bacteria of the IR population possessed a higher diversity and richness compared with the IS population. Furthermore, both the IS and IR populations were fed on the phloem sap and were maintained on the same rice variety in this study. Interestingly, we found variations in the composition of the gut bacteria between the two populations of $N$. lugens. The IR population exhibited a higher abundance of the phylum Firmicutes, whereas in the IS population, Proteobacteria was the most abundant. In the IR Plutella xylostella, Xia et al. [6] reported a similar trend of the higher abundance of Firmicutes compared with its susceptible counterparts. Furthermore, their study reported an increase in the proportion of the order Lactobacillales and a commensurate reduction in Enterobacteriaceae in P. xylostella resistant to fipronil and chlorpyrifos. In our study, comparisons at the order level showed that bacteria belonging to Lactobacillales and Enterobacteriales were significantly abundant in the IR population of $N$. lugens. The other bacterial orders significantly abundant in the IR population included Burkholderiales, which are known to perform the detoxification functions to their insect hosts and Clostridiales, which are involved in the breakdown of the lignocelluloses $[28,29]$. The Rhodospirillales and Rickettsiales, which were more abundant in the IS population, have been previously reported from many insect species [30,31].

In the present study, the differences in the composition of the gut bacteria between the IS and IR populations may be due to the adaptive responses of the microbiota to the 
differential gut environment, even though both the $\mathrm{BPH}$ populations were maintained in 20-25-day-old rice cv. TN1. Yun et al. [32] reported that diet is the controlling factor for the microbial diversity, and the presence of the higher numbers of bacterial diversity in the omnivorous insects compared with the carnivorous and herbivorous insects could be related to the types of food consumed. In this study, it may be hypothesized that as compared with the laboratory-reared susceptible population, the field population gets exposed to various stresses, including toxic compounds such as pesticides, which may impart a selection pressure on the insect in order to selectively enrich a bacterial community that can detoxify these xenobiotics [13]. The bacterial symbiont-mediated detoxification of the pesticide fenitrothion has been previously demonstrated by Kikuchi et al. [5] in the bean bug, Riptortus pedestris. They showed that experimental application of fenitrothion enriched the fenitrothion-degrading genus Burkholderia in the soil, which then colonized the midgut "crypts" of $R$. pedestris and conferred resistance of the host to the pesticide. The above results signify that the differences in the composition of the bacterial communities between the resistant and susceptible insects need to be identified at the genus or the species level. Therefore, we aimed to determine the abundance of the bacterial flora at the genus level and their putative functional profiles.

The abundant bacterial genera associated with the IS population included those belonging to the family Acetobacteraceae, all acetic acid-producing bacteria known to colonize the guts of several insect species and are reported to be the secondary symbionts of the Hemipterans and the Homopterans, relying on sugar-based diets and the phloem sap [30,33]. In the IR population, an unclassified genus, belonging to the order Bacteroidales, was found to be the most abundant. Another abundant genus, Trichococcus, has not been isolated previously from any insect. However, Trichococcus was isolated from the soil previously and was shown to possess xylanolytic activity [34].

Among the assigned genera (abundance count $\geq 100$ ) shared between the two populations, Morganella, Weissella, and Enterococcus may form the core bacterial genera associated with $N$. lugens. Furthermore, these genera were found to be more abundant in the IR population than in the IS population. All the three genera were previously reported from insect guts $[28,35]$. The gut-associated enterococci are known to contribute to the fitness of the insect host in several ways, including the prevention of proliferation of the pathogen [36, 37].
The other shared genera, including Prosthecochloris, Clostridiisalibacter, Fusibacter, Proteiniclasticum, Clostridium, and Bacillus, were found in higher abundance in the IR population than in the IS population. Among these, Clostridium and Bacillus sp. were found to be associated with several insect species, suggesting that these bacteria might perform the biologically relevant functions for their insect host [37-40]. Clostridia are the genera of obligate anaerobic bacteria and many of them are known to be pathogenic. However, owing to their ability to degrade polysaccharides into acetone, alcohol, acetate, and lactate, the gut-associated Clostridium is speculated to be involved in the nutritional physiology of the insect hosts [37]. Reportedly, Bacillus produces the digestive enzymes, including $\alpha$-amylase, cellulase, and xylanase, thereby providing nutritional benefits to their hosts. Furthermore, the various species of this genus are known for their ability to detoxify xenobiotics [40,41]. Gluconacetobacter, belonging to the Acetobacteraceae family, is among the shared genera that are more abundant in the IS population. Lactobacillus, Acinetobacter, Serratia, Pseudomonas, and Delftia were among the less predominant genera associated with both the populations. These genera are known to be frequently associated with the arthropod guts, possibly playing important roles in the fitness attribute of the host [37].

In the present study, the unique bacteria associated with the IS population belonged to the family Acetobacteraceae of the phylum Proteobacteria, whereas most of the unique bacteria associated with the IR population belonged to the phylum Firmicutes. The other relevant genera detected in the present study included Wolbachia and Arsenophonus. Previous studies showed that these two genera were among the endosymbiotic bacteria of the $\mathrm{BPH}$ that are maternally transmitted, and their occurrence might be exclusive to each individual host [16, 42]. A similar observation was made in our study, where we detected Wolbachia associated with the IS population, and Arsenophonus associated with the IR population. Furthermore, many rarely abundant genera were found to be associated with the metagenome of the IR population in our study. Understanding the roles played by these rarely abundant genera will enable the elucidation of their possible roles in the nutrition, detoxification, and overall fitness of the host.

The variations in the composition of the gut-associated bacteria between the IS and IR populations may reflect the distinctive differences in the functional requirements between the two populations. The taxonomic abundance data were subjected to phenotypic mapping to obtain the 
putative functional profiles. Functional predictions based on taxonomic data using METAGENassist have been previously demonstrated for associated bacteria from various ecological niches, including the earthworm gut [43]. The microbes promote the utilization of the diets that are low in nitrogen by fixing the atmospheric nitrogen as well as by utilizing the nitrogenous waste products produced by their host and synthesizing the essential amino acids out of them [44]. The upregulation of nitrogen fixation in the guts of the IR population might be attributed to the higher numerical abundance of Enterobacteriaceae, a member of diazotrophic bacteria, which enable nitrogen fixation [17]. The association of $N$. lugens with the bacterial flora involved in the metabolism of nitrogen might enable the host to survive on a nutrient-deficient diet such as the phloem sap [45]. The involvement of the gut-associated bacteria in sulfur cycling, as evidenced by the presence of the sulfate-reducing bacterium Desulfovibrio, has been previously implicated in other insects, including the termite Mastotermes darwiniensis [46]. The phloem is found to be rich in cellulose, hemicellulose, and soluble carbohydrates [47]. Thus, the cellulose and xylan degraders in the gut metagenome of $N$. lugens, including those belonging to the order Clostridiales such as the genus Butyrivibrio, might be involved in the digestion of the dietary lignocellulose to sugars [48]. Indiragandhi et al. [49] reported that chitinaseproducing gut bacteria increased the host insect consumption index, relative growth rate, and approximate digestibility in P. xylostella. Chitin-degrading bacteria associated with the gut of $N$. lugens might be performing similar functions for its host. Furthermore, it was found that the IR population was enriched with bacteria involved in the degradation of the aromatic hydrocarbons and alkanes, and the metabolism of atrazine. The selective enrichment of these bacteria involved in such detoxifying mechanisms can be an adaptive response of the IR population in order to survive the toxic compounds, including the plant allelochemicals and synthetic compounds such as pesticides that are encountered in the field. It is striking that Bacillus sp., known for its ability to degrade organophosphate insecticides including malathion, are abundant in the IR population, presumably indicating the functional adaptation by the IR population by altering the gut bacteria [41, 50]. It was previously reported that Bacillus spp. degrade organophosphate pesticides through the enzyme activity of carboxyl esterases [51]. Furthermore, the genus Pseudomonas, capable of degrading xenobiotics such as insecticides, was detected in the present study [52]. The associated bacteria have been suggested to contribute to the enzymatic hydrolysis of insecticides in the insect gut, which in turn may provide nutrients for the bacterial flora [13]. van den Bosch and Welte [53] suggested the term "detoxifying symbiosis," where the microorganisms, associated with the insect mediate the detoxification of plant toxins and insecticides [53]. The possibility of such an association between the IR population and its bacterial flora needs to be further investigated such that novel strategies of pest control can be formulated.

In conclusion, through deep sequencing of the V3 hypervariable region of the $16 \mathrm{~S}$ rRNA gene, a comparative catalog of the bacterial communities associated with the guts of the IR and IS populations of $N$. lugens was presented. Overall, the N. lugens gut-associated bacteria are capable of performing diverse functions, thereby contributing to the various fitness attributes of the host. The IR field population was found to be associated with diverse bacterial communities and was enriched with bacteria capable of performing detoxification-related functions. However, it is important to ascertain a thorough functional characterization of the symbionts to elucidate their possible roles in the detoxification of insecticides. Such studies will enable the implementation of novel strategies to control the insect pests by manipulating their gut bacteria. The present study is the first attempt to catalog the gut bacteria of N. lugens by the deep-sequencing approach. The bacteria identified in the present study can form the basis for future studies on symbiont-based strategies to manage the resistance to insecticides in N. lugens.

\section{Acknowledgments}

This work was supported by the Strategic Initiative for Microbiomes in Agriculture and Food, Ministry of Agriculture (914004-4), Food and Rural Affairs, Republic of Korea. We are also thankful to the Director, ICAR-National Bureau of Agricultural Insect Resources, Bengaluru, India for providing necessary laboratory facilities. VMM gratefully acknowledges the Department of Science and Technology, Government of India for providing an INSPIRE doctoral fellowship. This work is a part of the doctoral thesis of VMM registered under the University of Mysore, India.

\section{Conflict of Interest}

The authors have no financial conflicts of interest to declare. 


\section{References}

1. Garrood WT, Zimmer CT, Gorman KJ, Nauen R, Bass C, Davies TG. 2015. Field-evolved resistance to imidacloprid and ethiprole in populations of brown planthopper Nilaparvata lugens collected from across South and East Asia. Pest Manag. Sci. 72: 140-149.

2. Min S, Lee SW, Choi BR, Lee SH, Kwon DH. 2014. Insecticide resistance monitoring and correlation analysis to select appropriate insecticides against Nilaparvata lugens (Stal), a migratory pest in Korea. J. Asia Pac. Entomol. 17: 711-716.

3. Puinean AM, Denholm I, Millar NS, Nauen R, Williamson MS. 2010. Characterisation of imidacloprid resistance mechanisms in the brown planthopper, Nilaparvata lugens Stål (Hemiptera: Delphacidae). Pest Biochem. Physiol. 97: 129-132.

4. Hemingway J, Karunaratne SH. 1998. Mosquito carboxylesterases: a review of the molecular biology and biochemistry of a major insecticide resistance mechanism. Med. Vet. Entomol. 12: 1-12.

5. Kikuchi Y, Hayatsu M, Hosokawa T, Nagayama A, Tago K, Fukatsu T. 2012. Symbiont-mediated insecticide resistance. Proc. Natl. Acad. Sci. USA 109: 8618-8622.

6. Xia X, Zheng D, Zhong H, Qin B, Gurr GM, Vasseur L, et al. 2013. DNA sequencing reveals the midgut microbiota of diamondback moth, Plutella xylostella (L.) and a possible relationship with insecticide resistance. PLoS One 8: e68852.

7. Kikuchi Y. 2009. Endosymbiotic bacteria in insects: their diversity and culturability. Microbes Environ. 24: 195-204.

8. Engel P, Moran NA. 2013. The gut microbiota of insects diversity in structure and function. FEMS Microbiol. Rev. 37: 699-735.

9. Jones RT, Bressan A, Greenwell AM, Fierer N. 2011. Bacterial communities of two parthenogenetic aphid species cocolonizing two host plants across the Hawaiian islands. Appl. Environ. Microbiol. 77: 8345-8349.

10. Warnecke F, Luginbühl P, Ivanova N, Ghassemian M, Richardson TH, Stege JT, et al. 2007. Metagenomic and functional analysis of hindgut microbiota of a wood-feeding higher termite. Nature 450: 560-565.

11. Lin XL, Pan QJ, Tian HG, Douglas AE, Liu TX. 2015. Bacteria abundance and diversity of different life stages of Plutella xylostella (Lepidoptera: Plutellidae), revealed by bacteria culture-dependent and PCR-DGGE methods. Insect Sci. 22: 375-385.

12. Cheng D, Guo Z, Riegler M, Xi Z, Liang G, Xu Y. 2017. Gut symbiont enhances insecticide resistance in a significant pest, the orient fruit fly Bactrocera dorsalis (Hendel). Microbiome 5: 13.

13. Almeida LG, Moraes LA, Trigo JR, Omoto C, Cônsoli FL. 2017. The gut microbiota of insecticide-resistant insects houses insecticide degrading bacteria: a potential source for biotechnological exploitation. PLoS One 12: e0174754.

14. Gayatri Priya N, Ojha A, Kajla MK, Raj A, Rajagopal R. 2012. Host plant induced variation in gut bacteria of Helicoverpa armigera. PLoS One 7: e30768.
15. Tagliavia M, Messina E, Manachini B, Cappello S, Quatrini P. 2014. The gut microbiota of larvae of Rhynchophorus ferrugineus Oliver (Coleoptera: Curculionidae). BMC Microbiol. 14: 136.

16. Tang M, Lv L, Jing SL, Zhu LL, He GC. 2010. Bacterial symbionts of the brown planthopper, Nilaparvata lugens (Homoptera: Delphacidae). Appl. Environ. Microbiol. 76: 1740-1745.

17. Wang A, Yao Z, Zheng W, Zhang H. 2014. Bacterial communities in the gut and reproductive organs of Bactrocera minax (Diptera: Tephritidae) based on 454 pyrosequencing. PLoS One 9: e106988.

18. Malathi VM, Jalali SK, Sidde Gowda DK, Mohan M, Venkatesan T. 2015. Establishing the role of detoxifying enzymes in field-evolved resistance to various insecticides in the brown planthopper, Nilaparvata lugens in South India. Insect. Sci. 24: 35-46.

19. Kim YJ, Lee YJ, Kim GH, Lee SW, Ahn YJ. 1999. Toxicity of tebufenpyrad to Tetranychus urticae (Acari: Tetranychidae) and Amblyseius womersleyi (Acari: Phytoseiidae) under laboratory and field conditions. J. Econ. Entomol. 92: 187-192.

20. Bartam AK, Lynch MDJ, Stearns JC, Moreno-Hagelsieb G, Nuefeld JD. 2011. Generation of multimillion-sequence $16 S$ rRNA gene libraries from complex microbial communities by assembling paired-end Illumina reads. Environ. Microbiol. 77: 3846-3852.

21. Caporaso JG, Kuczynski J, Stombaugh J, Bittinger K, Bushman FD, Costello EK, et al. 2010. QIIME allows analysis of high-throughput community sequencing data. Nat. Methods 7: 335-336.

22. Parks DH, Tyson GW, Hugenholtz P, Beiko RG. 2014. STAMP: statistical analysis of taxonomic and functional profiles. Bioinformatics 30: 3123-3124.

23. Arndt D, Xia J, Liu Y, Zhou Y, Guo AC, Cruz JA, et al. 2012. METAGENassist: a comprehensive web server for comparative metagenomics. Nucleic Acids Res. 40: W88-W95.

24. Metsalu T, Vilo J. 2015. ClustVis: a web tool for visualizing clustering of multivariate data using Principal Component Analysis and heatmap. Nucleic Acids Res. 43: W566-W570.

25. Wang Y, Naumann U, Wright ST, Warton DI. 2012. Mvabund - an $\mathrm{R}$ package for model-based analysis of multivariate abundance data. Methods Ecol. Evol. 3: 471-474.

26. Team RC. 2014. R: a language and environment for statistical computing [Internet]. R Foundation for Statistical Computing, Vienna, Austria.

27. Xu HX, Zheng XS, Yang YJ, Wang X, Ye GY, Lu ZX. 2014. Bacterial community in different populations of rice brown planthopper, Nilaparvata lugens (Stål). Rice Sci. 21: 59-64.

28. Zhang J, Zhang Y, Li J, Liu M, Liu Z. 2016. Midgut transcriptome of the cockroach Periplaneta americana and its microbiota: digestion, detoxification and oxidative stress response. PLoS One 1: e0155254.

29. Boucias DG, Cai Y, Sun Y, Lietze V-U, Sen R, Raychoudhury R, et al. 2013. The hindgut lumen prokaryotic microbiota of the termite Reticulitermes flavipes and its responses to dietary 
lignocellulose composition. Mol. Ecol. 22: 1836-1853.

30. Chandler JA, Morgan Lang J, Bhatnagar S, Eisen JA, Kopp A. 2011. Bacterial communities of diverse Drosophila species: ecological context of a host-microbe model system. PLoS Genet. 7: e1002272.

31. Kautz S, Rubin BER, Moreau CS. 2013. Bacterial infections across the ants: frequency and prevalence of Wolbachia, Spiroplasma, and Asaia. Psyche (Camb. Mass.) 2013: 936341.

32. Yun J-H, Roh SW, Whon TW, Jung M-J, Kim M-S, Park D-S, et al. 2014. Insect gut bacterial diversity determined by environmental habitat, diet, developmental stage, and phylogeny of host. Appl. Environ. Microbiol. 80: 5254-5264.

33. Segata N, Baldini F, Pompon J, Garrett WS, Truong DT, Dabiré RK, et al. 2016. The reproductive tracts of two malaria vectors are populated by a core microbiome and by gender and swarm-enriched microbial biomarkers. Sci. Rep. 6: 24207.

34. Gao ZM, Xu X, Ruan LW. 2014. Enrichment and characterization of an anaerobic cellulolytic microbial consortium SQD-1.1 from mangrove soil. Appl. Microbiol. Biotechnol. 98: 465-474.

35. Ramya SL, Venkatesan T, Srinivasa Murthy K, Jalali SK, Verghese A. 2015. Detection of carboxylesterase and esterase activity in culturable gut bacterial flora isolated from diamondback moth, Plutella xylostella (Linnaeus), from India and its possible role in indoxacarb degradation. Braz. J. Microbiol. 47: 327-336.

36. van Frankenhuyzen K, Liu Y, Tonon A. 2010. Interactions between Bacillus thuringiensis subsp. kurstaki HD-1 and midgut bacteria in larvae of gypsy moth and spruce budworm. J. Invertebr. Pathol. 103: 124-131

37. Chen B, The BS, Sun C, Hu S, Lu X, Boland W, et al. 2016. Biodiversity and activity of the gut microbiota across the life history of the insect herbivore Spodoptera littoralis. Sci. Rep. 6: 29505.

38. Tokuda G, Yamaoka I, Noda H. 2000. Localization of symbiotic clostridia in the mixed segment of the termite Nasutitermes takasagoensis (Shiraki). Appl. Environ. Microbiol. 66: 2199-2207.

39. Tang X, Freitak D, Vogel H, Ping L, Shao Y, Cordero EA, et al. 2012. Complexity and variability of gut commensal microbiota in polyphagous lepidopteran larvae. PLoS One 7: e36978.

40. Snyman M, Gupta AK, Bezuidenhout CC, Claassens S, van den Berg J. 2016. Gut microbiota of Busseola fusca (Lepidoptera: Noctuidae). World J. Microbiol. Biotechnol 32: 115.

41. Pemberton JM, Wynn EC. 1984. Genetic engineering and biological detoxification/degradation of insecticides, pp. 147168. In Lal R (ed.), Insecticide Microbiology. Springer, Berlin.

42. Qu LY, Lou YH, Fan HW, Ye YX, Huang HJ, Hu MQ, et al. 2013. Two endosymbiotic bacteria, Wolbachia and Arsenophonus, in the brown planthopper Nilaparvata lugens. Symbiosis 61: 47.

43. Singh A, Singh DP, Tiwari R, Kumar K, Singh RV, Singh S, et al. 2015. Taxonomic and functional annotation of gut bacterial communities of Eisenia foetida and Perionyx excavatus. Microbiol. Res. 175: 48-56.

44. Douglas AE. 2015. Multiorganismal insects: diversity and function of resident microorganisms. Annu. Rev. Entomol. 60: $17-34$.

45. Dillon RJ, Dillon VM. 2004. The gut bacteria of insects: nonpathogenic interactions. Annu. Rev. Entomol. 49: 71-92.

46. Dröge S, Limper U, Emtiazi F, Schönig I, Pavlus N, Drzyzga O, et al. 2005. In vitro and in vivo sulfate reduction in the gut contents of the termite Mastotermes darwiniensis and the rosechafer Pachnoda marginata. J. Gen. Appl. Microbiol. 51: 57-64.

47. Morales-Jimenez J, Zuniga G, Villa-Tanaca L, HernandezRodriguez C. 2009. Bacterial community and nitrogen fixation in the red turpentine beetle, Dendroctonus valens LeConte (Coleoptera: Curculionidae: Scolytinae). Microb. Ecol. 58: 879-891.

48. Köhler T, Dietrich C, Scheffrahn RH, Brune A. 2012. Highresolution analysis of gut environment and bacterial microbiota reveals functional compartmentation of the gut in wood-feeding higher termites (Nasutitermes spp.). Appl. Environ. Microbiol. 78: 4691-4701.

49. Indiragandhi P, Anandham R, Madhaiyan M, Kim GH, Sa TM. 2008. Cross utilization and expression of outer membrane receptor proteins for siderophores uptake by diamondback moth Plutella xylostella (Lepidoptera: Plutellidae) gut bacteria. FEMS Microbiol. Lett. 287: 27-33.

50. Singh B, Singh K. 2016. Bacillus: as bioremediator agent of major environmental pollutants, pp. 35-55. In Islam MT, Rahman M, Pandey P, Jha CK, Aeron A (eds.). Bacilli and Agrobiotechnology. Springer, Berlin.

51. Ramya SL, Venkatesan T, Srinivasa Murthy K, Jalali SK, Verghese A. 2016. Detection of carboxylesterase and esterase activity in culturable gut bacterial flora isolated from diamondback moth, Plutella xylostella (Linnaeus), from India and its possible role in indoxacarb degradation. Braz. J. Microbiol. 47: 327-336.

52. Indiragandhi $\mathrm{P}$, Anandham R, Madhaiyan M, Poonguzhali S, Kim GH, Saravanan VS, et al. 2007. Cultivable bacteria associated with larval gut of prothiofos-resistant, prothiofossusceptible and field-caught populations of diamondback moth, Plutella xylostella and their potential for, antagonism toward entomopathogenic fungi and host insect nutrition. J. Appl. Microbiol. 103: 2664-2675.

53. van den Bosch TJ, Welte CU. 2016. Detoxifying symbionts in agriculturally important pest insects. Microb. Biotechnol. 10: 531-540. 